Conclusions In our cohort of $167,62 \%(n=104)$ of patients had a TL below the recently published recommended guidelines $(5 \mu \mathrm{g} / \mathrm{ml})$ yet were in a clinical remission. Therefore following these guidelines would lead to a significant increase in drug spend which may not translate into improved clinical outcomes; since in this cohort only $24 \%$ with sub-therapeutic levels had active disease. Escalating only those with active disease may represent a more acceptable financial solution but too will lead to an increase in drug spend.

\section{PWE-019 DAY OF ADMISSION RESULTS PREDICT OUTCOME IN ACUTE ULCERATIVE COLITIS}

${ }^{1}$ lan Arnott*, ${ }^{2}$ Ruairi Lynch, ${ }^{3}$ Thomas Manship, ${ }^{1}$ Fiona Jagger, ${ }^{1}$ Jack Satsangi, ${ }^{1} G$ woTzer Ho, ${ }^{1}$ Charlie Lees, ${ }^{2}$ MissRebecca Grant. 'Western General Hospital, Edinburgh, UK; ${ }^{2}$ University of Edinburgh, Edinburgh, UK; ${ }^{3}$ Royal Infirmary of Edinburgh, Edinburgh, UK

\subsection{6/gutjnl-2018-BSGAbstracts.151}

Introduction Intravenous steroids remain the standard first line treatment for patients with acute ulcerative colitis (UC). However, $30 \%$ of patients fail to respond and require second line therapies and/or surgery. The purpose of this study was to determine if day 1 parameters could identify a group at high risk of failing first line therapies.

Methods All admissions for acute UC (ICD-10 K51) to hospitals within NHS Lothian $\left(4\right.$ sites) from $1^{\text {st }}$ November 2015 to $31^{\text {st }}$ October 2016 were obtained from the regional coding department. Inclusion was limited to patients with UC as the primary reason for admission. Case record review confirmed diagnosis and clinical data were collected. Response to steroids was defined as discharge from hospital with no further acute medical or surgical treatment. The following parameters were recorded up to the first 10 days post admission: haemoglobin, platelet count, CRP, albumin, stool frequency, faecal calprotectin and abdominal $\mathrm{x}$-ray results. Each patient was later attributed a score based on CRP $(<50 \mathrm{mg} / \mathrm{dL}=0 ;>50 \mathrm{mg} / \mathrm{dL}=1)$, albumin $(>30 \mathrm{~g} / \mathrm{L}=0 ;<30 \mathrm{~g} / \mathrm{L}=1)$ and platelets $\left(<450 \times 10^{9}\right)$ $\left.\mathrm{L}=0 ;>450 \times 10^{9} / \mathrm{L}=1\right)$.

Results From 1 st November 2015 to 31 st October 201661 admissions with acute UC were identified; 37 (60\%) of which responded to steroids, $24(40 \%)$ patients were non-responders. $3(12.5 \%)$ non-responders received infliximab as second line therapy, 1 (4.2\%) adalimumab and $20(83.3 \%)$ ciclosporin; 11 $(45.8 \%)$ of the non-responders required surgery, $100 \%$ of whom had been previously treated with ciclosporin. On univariate analysis, the albumin, platelet count and CRP differed significantly between responders and non-responders $(p \leq 0.05)$, whereas on multivariate analysis only CRP and albumin were significant. No difference was seen for haemoglobin and stool frequency. $88.9 \%$ of patients with concurrent hypoalbuminaemia, high CRP and high platelets $($ score $=3)$ were nonresponders.
Conclusions $82.4 \%$ of patients with a score of 2 or more will fail first line medical therapy. The combination of these readily available parameters identifies a high-risk population who may benefit from earlier second line medical or surgical intervention.

\begin{tabular}{|c|c|c|c|}
\hline Total score & No. of patients & Responders (\%) & Non-responders (\%) \\
\hline $0-1$ & 29 & $23(79.3)$ & $6(20.7)$ \\
\hline $2-3$ & 17 & $3(17.6)$ & $14(82.4)$ \\
\hline
\end{tabular}

\section{PWE-020 INITIAL EXPERIENCE OF A RAPID ACCESS ULTRASOUND IMAGING CLINIC IN INFLAMMATORY BOWEL DISEASE}

Nigel Grunshaw*, Francis Egbuonu, Wendi Harrison, Albert Davies. University Hospitals Morecambe Bay NHS Trust, Barrow In Furness, UK

\subsection{6/gutjnl-2018-BSGAbstracts. 152}

Introduction Although less widely used than MRI in UK practice, Ultrasound offers a number of advantages in the assessment of inflammatory bowel disease. It is quick to perform and requires minimal preparation allowing it to be performed at short notice. It is dynamic and clinically interactive allowing immediate correlation of patient's symptoms with imaging findings. It provides assessment of disease activity/complications together with dynamic assessment of functional obstruction. Results are immediately available allowing instantaneous clinical decision making. We present our initial experience of using Ultrasound in a rapid access imaging clinic and its effects on patient management.

Methods Patients were referred from either clinic, endoscopy or via IBD specialist nurse direct access/telephone advice line. Dedicated slots were allocated on Ultrasound lists, and appointments directly booked by the IBD nurse. All referrals were made on the basis of possible new diagnosis of IBD, assessment of disease extent following endoscopic diagnosis or known patient with IBD with possible exacerbation/complications. All patients were scanned by a single Consultant GI Radiologist in the presence of the IBD specialist nurse. Findings were immediately communicated to patient and nurse and immediate combined decision made with regard to necessity for treatment change and urgency of further follow up.

Results 54 patients were examined: - Male: Female 21:33. Age range $17-79$ years. $48 \%$ of patients were examined within 7 days and 69\% within 14 days of referral. Ultrasound detected active disease/complications in $65 \%$. Ultrasound findings resulted $43 \%$ of patients undergoing significant change in management with $41 \%$ also requiring dietetic referral. $29 \%$ required urgent follow up within 1 week including 3 patients

Abstract PWE-019 Table 1 Day one results

\begin{tabular}{|c|c|c|c|c|c|c|}
\hline & \multicolumn{2}{|c|}{ Platelets $\left(\times 10^{9} / \mathrm{L}\right)$} & \multicolumn{2}{|c|}{ CRP (mg/dL) } & \multicolumn{2}{|c|}{ Albumin ( $g / L)$} \\
\hline & $\begin{array}{l}\text { Responders } \\
\qquad(n=36)\end{array}$ & Non-responders $(n=23)$ & $\begin{array}{c}\text { Responders } \\
(\mathrm{n}=32)\end{array}$ & $\begin{array}{l}\text { Non-responders } \\
\qquad(\mathrm{n}=22)\end{array}$ & $\begin{array}{c}\text { Responders } \\
\quad(n=27)\end{array}$ & $\begin{array}{c}\text { Non-responders } \\
(n=23)\end{array}$ \\
\hline Median & 342 & 464 & 25 & 78 & 34 & 27 \\
\hline Interquartile range & $107(291-398)$ & $172(345-517)$ & $45(9-54)$ & $131(44-175)$ & $5(31-36)$ & $9(24-33)$ \\
\hline
\end{tabular}


that required immediate Consultant review after the scan. 9 patients (17\%) required further imaging with CT/MR during the course of their follow up.

Conclusions Our experience demonstrates that using a combined multi-disciplinary clinico- radiological approach with Ultrasound as the primary imaging modality in IBD offers a number of advantages. These include rapid access and flexibility of imaging appointments, minimal patient preparation and instantaneous availability of results allowing immediate feedback and patient counselling. It provides the opportunity for urgent treatment changes and triage of follow up appointment scheduling. Wider adoption of Ultrasound in IBD has the potential to offer significant improvement in speed of diagnosis and patient management.

\section{PWE-021 LOCAL EXPERIENCE OF VSL\#3 USE IN POUCHITIS}

James Gulliver*, Stephen Lewis, Walter Douie. Plymouth Hospitals NHS Trust, UK

\subsection{6/gutjnl-2018-BSGAbstracts. 153}

Introduction Pouchitis can affect up to $50 \%$ of patients following ileal pouch-anal anastomosis (IPAA). Broad spectrum antibiotics are the mainstay of treatment. In 2003 a randomised study of 36 patients showed remission rates of $85 \%$ at 1 year with the probiotic VSL\#3, ${ }^{1}$ In 2005 a US study of 31 patients in clinical practice failed to replicate these results. ${ }^{2} \mathrm{~A}$ Cochrane review found low quality evidence to support the use of VSL\#3 in maintaining remission for chronic pouchitis. ${ }^{3}$ We retrospectively evaluated the use of VSL\#3 in our teaching hospital, to see if the efficacy demonstrated in the earlier study could be replicated.

Methods We interrogated our surgical database for patients undergoing IPAA between 2003 and 2014. Paper and electronic records were reviewed for details of pouchitis episodes, their investigation and subsequent management. Diagnosis was classified as probable with clinical evaluation only, or definitive based on histological confirmation. Results were tabulated, analysed non-parametrically and presented as medians and interquartile ranges.

Results Pouchitis was diagnosed in $27 \%$ of IPAA patients. This cohort had an average age of 48 years and a female preponderance of 1.2:1. The median duration to first episode was 43 months (34-75). The diagnosis was probable in $29 \%$ and definitive in $71 \%$ of patients. Antibiotics (metronidazole in 100\%, ciprofloxacin in 13\%) were prescribed in $66.7 \%$ of patients.

14 patients $(58 \%)$ were commenced on VSL\#3 following their pouchitis (figure 1). Of those with confirmed recurrent pouchitis, two thirds remain on VSL\#3 but use repeated antibiotic courses to settle flares. The median duration of follow up was 92.5 months (48-75).

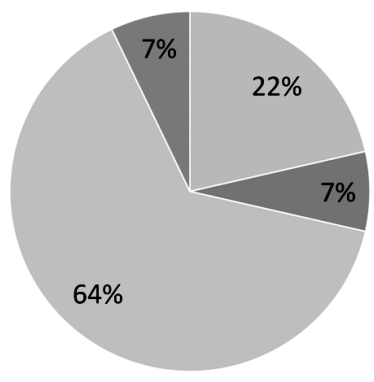

No evidence of
further pouchitis
Clinical diagnosis
recurrent pouchitis
Histologically
confirmed recurrent
pouchitis

- VSL\#3 discontinued

Abstract PWE-021 Figure 1 Outcomes of VSL\#3 use after first episode of pouchitis
Conclusions This study evaluated the use of VSL\#3 over a longer follow up than those published previously. VSL\#3 was efficacious in only $22 \%$ of the cohort. Accepting the limitations of this small study, the data suggests VSL\#3 has little effect in maintaining remission in pouchitis post IPAA. These results correlate with the earlier US study where $19 \%$ of patients demonstrated efficacy at 8 months. Larger studies are recommended to review the benefits of VSL\#3 in this cohort, with specific reference to degrees of severity and numbers of previous episodes of pouchitis.

\section{REFERENCES}

1. Mimura, et al. Once daily high dose probiotic therapy (VSL\#3) for maintaining remission in recurrent or refractory pouchitis. Gut 2004;53:108-114.

2. Shen, et al. Maintenance therapy with a probiotic in antibiotic-dependent pouchitis: experience in clinical practice. Aliment Pharmacol Ther. 2005 Oct 15;22 (8):721-8.

3. Holubar, et al. Treatment and prevention of pouchitis after ileal-pouch anal anastomosis for ulcerative colitis. Cochrane Database Syst Rev 2010;6:CD001176

\section{PWE-022 GUT-HOMING TH17 CELLS ARE SELECTIVELY TARGETED BY VEDOLIZUMAB AND MAY PREDICT CLINICAL RESPONSE IN IBD}

Sherine Hermangild Kottoor*, Cristiano Scotta, Polychronis Pavlidis, Ellie Alberts, Zain Kassam, Laura Constable, Michael Metoudi, Jonathan Digby-Bell, Hajir Ibraheim, Dawn Warren, Mark Samaan, Peter Irving, Jeremy Sanderson, Nick Powell. Kings Health Partners, UK

\subsection{6/gutjnl-2018-BSGAbstracts. 154}

Introduction Trafficking of inflammatory lymphocytes to the gut plays a central role in IBD pathogenesis. We analysed the profile of circulating gut homing effector memory $\mathrm{T}$ cell subsets in IBD patients. We also evaluated the impact of treatment with Vedolizumab, a monoclonal antibody that binds to integrin $\alpha_{4} \beta_{7}(\beta 7)$ and prevents binding to its ligand MAdCAM-1, thereby preventing lymphocyte migration to the gut.

Methods Using multi parametric flow cytometry, we analysed the gut homing $(\beta 7+)$ effector T-cells $\left(\mathrm{CD}^{+}{ }^{+} \mathrm{CD}^{+}{ }^{+} \mathrm{CD} 45 \mathrm{RO}^{+} \mathrm{CD} 4 \mathrm{RA}^{-} \mathrm{CCR} 7^{-}\right)$including different functional lineages: Th1 $\left(\mathrm{CXCR}^{+}{ }^{+} \mathrm{CCR} 6^{-}\right)$; $\mathrm{Th} 2$

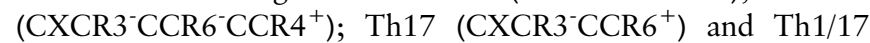
$\left(\mathrm{CXCR}^{+}{ }^{+} \mathrm{CCR} 6^{+}\right)$from peripheral blood $(\mathrm{PB})$ of healthy controls $(\mathrm{HC}, \mathrm{n}=42)$ and IBD $(\mathrm{n}=34)$ patients, including a prospective analysis of new starters of vedolizumab. Peripheral blood was taken from patients before their first dose of vedolizumab and at each subsequent infusion.

Results Compared to HC, the proportion of Th1 cells within the gut homing compartment was significantly decreased in $\mathrm{PB}$ of IBD patients (median HC 27.3\% vs IBD 44\%, p<0.0006). In contrast, the proportion of Th17 cells within the gut homing compartment was significantly increased (HC $12 \%$ vs IBD $19 \%, \mathrm{p}<0.003)$. This difference was most striking in ulcerative colitis. There was no significance difference in Th1/17 or Th2 cells in IBD vs HC.

In the longitudinal analysis, there was minimal impact on gut homing Th1 cells in vedolizumab treated patients (comparison between baseline and week 8), however, the gut homing Th17 compartment increased over the same time period (from $19.3 \%$ at baseline to $29.7 \%$ at week 8 ). The proportion of gut homing Th17 was significantly higher in vedolizumab treated patients at week 8 in comparison to infliximab $(n=3)$ 